\title{
Protée
}

\section{La danse ou la mort : l'art de la terreur sans le sang}

\section{Liora Malka}

Volume 27, numéro 1, 1999

La Mort de Molière et des autres

URI : https://id.erudit.org/iderudit/030540ar

DOI : https://doi.org/10.7202/030540ar

Aller au sommaire du numéro

\section{Éditeur(s)}

Département des arts et lettres - Université du Québec à Chicoutimi

\section{ISSN}

0300-3523 (imprimé)

1708-2307 (numérique)

Découvrir la revue

\section{Citer cet article}

Malka, L. (1999). La danse ou la mort : l'art de la terreur sans le sang. Protée, 27(1), 47-52. https://doi.org/10.7202/030540ar

\section{Résumé de l'article}

La vidéo de Robert Wilson, La Mort de Molière, contient quatre scènes où l'action peut être identifiée à une danse. Bien qu'une danse communique toujours l'image d'un corps humain en mouvement, cette production de Wilson génère une distinction et, par la suite, une interaction entre un corps dansant et d'autres images du corps, celles entre autres du corps de Molière mourant. Mon analyse porte plus spécifiquement sur ces interrelations entre le corps dansant et le corps mourant, afin de mieux comprendre celles entre le monde fictionnel de Molière et l'univers esthétique de Wilson. 


\section{LA DANSE OU LA MORT: L'ART DE LA TERREUR SANS LE SANG}

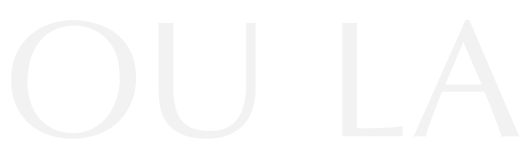

Traduit de I'anglais par Jean-Pierre Vidal

LIORA MALKA

La vidéo La Mort de Molière de Robert Wilson est construite comme un collage fragmenté d'images et d'actions interactives qui semblent à première vue ne pas avoir de rapport direct. Même si l'œuvre présente un univers fictionnel - la vie et la mort de Molière -, il n'y a pas d'intrigue suivie et les fragments sont liés entre eux plus par association que par une certaine continuité diégétique. Aussi la vidéo apparaît-elle ouverte à diverses formes de perception et de compréhension et aucun sens global ne semble émerger. Mais l'œuvre n'en dégage pas moins une impression de cohérence quant à sa composition esthétique. Elle instaure donc une contradiction dynamique entre l'univers fictionnel représenté, mettant en jeu Molière, et l'univers esthétique présenté, celui de Wilson.

La vidéo contient plusieurs scènes présentant des performances artistiques, comme la danse et la musique. Toutes ces scènes se déroulent sur une surface carrée blanche qui, dans cette œuvre, représente la scène de l'art et semble illustrer la contradiction dynamique entre l'univers de la fiction et sa présentation esthétique. La danse est de toutes ces scènes artistiques celle qui revient le plus souvent et elle constitue donc un important motif de l'œuvre. La vidéo contient quatre scènes dans lesquelles on peut nettement assimiler l'action à de la danse: la danse de Louis XIV (section 2); la danse des deux acteurs en noir et blanc (section 3); la danse du jeune garçon et de la jeune fille (section 5); et la danse du renard (section 7). Même si la danse véhicule toujours une image du corps humain, l'œuvre de Wilson établit une distinction et une interaction entre le corps qui danse et d'autres images du corps, en particulier du corps de Molière mourant. Mon analyse portera sur ces interrelations entre le corps dansant et le corps mourant et tentera d'élucider les relations interactives, mentionnées cidessus, entre l'univers représenté par la fiction, celui de Molière, et l'univers esthétique de Wilson.

Dès le début s'installe une contradiction entre «le corps du roi pris par la danse» et le corps de Molière "saisi par la douleur». Molière est couché dans son lit; sa bouche grande ouverte et hurlante dans la première séquence et les mouvements convulsifs de son corps dans la deuxième indiquent l'agonie et la douleur. La présentation du corps dans la douleur, qui instaure la situation 
dramatique fondamentale, représente ainsi l'étreinte de la mort et ses effets sur le corps humain, ce qui à son tour évoque l'emprise et le pouvoir du temps sur le corps et la vie de l'homme.

Les plans qui montrent les mouvements convulsifs du corps de Molière constituent la seule séquence dans laquelle son corps bouge dans son ensemble. À partir de là et tout au long de la vidéo, nous ne verrons plus bouger que des parties de son corps, et particulièrement sa bouche et ses mains. Bien que les parties du corps soient généralement considérées comme des métonymies du corps dans son ensemble, c'est ici plutôt une image de disparition qui se trouve convoquée: considéré dans son ensemble, le corps de Molière n'est plus en effet qu'une entité absente. Une dualité de l'être - des parties du corps agissantes - et du non-être - le corps complet dans son absence travaille ainsi l'œuvre, constituant un thème central qui renvoie à Molière comme à une identité subjective historique changée en une entité culturelle anhistorique. Une telle dualité réfère à la situation paradoxale qui consiste à être mort tout en étant vivant et elle inscrit le travail de la vidéo lui-même au cœur de ce paradoxe. Ainsi Wilson place-t-il son œuvre dans une situation limite et ironique, un entredeux de l'art et de la vie, de la culture et de l'histoire, de l'expérience et de la mémoire.

\section{LA DANSE DU ROI}

Immédiatement après les mouvements convulsifs de Molière, la caméra entame une nouvelle scène dans laquelle on voit Louis XIV d'abord écouter lire Racine, puis danser. La représentation du roi en train de danser tandis que Molière se meurt constitue un contraste cynique, qui renforce les images précédentes du corps perclus de douleurs. Le corps du roi se déplace librement dans un espace ouvert; la danse souligne la verticalité du corps et le contrôle qu'il a de ses mouvements, montrés par une chorégraphie esthétisante comme une suite de transitions d'une posture à une autre. Le corps de Molière, par contraste, est en position horizontale, recouvert d'un drap et cloué à l'espace restreint du lit. De plus,
Molière n'a aucun contrôle de ses mouvements: ses convulsions donnent l'impression d'être des mouvements spontanés et incontrôlables causés par la douleur et liés à la maîtrise qu'exerce le temps sur le corps. Ainsi se marquent, au niveau du mouvement, un ensemble d'oppositions entre le naturel et le spontané d'une part, l'artificiel et le formel d'autre part; de même qu'entre un corps contrôlé par le temps et un corps libre dans l'espace. Ces contradictions, qui représentent la différence entre vivre et mourir, se trouvent encore renforcées par la présentation tout en contraste de la vie et de l'art. Et en dépit de ces contradictions une ironie paradoxale vient jouer obscurément à plusieurs niveaux à la fois.

Dans le contexte de la vidéo comme œuvre d'art, les mouvements que la douleur arrache à Molière ne sont qu'une représentation artistique et sont donc foncièrement aussi artificiels et formels que les mouvements de danse. La distinction entre la vie et l'art s'en trouve ainsi brouillée au profit de la vision esthétique de Wilson qui nous fait éprouver tout l'art, toute l'habileté, de la vie et de la mort. De plus, la distinction entre le corps dans le temps et le corps dans l'espace vient contredire la condition naturelle du corps humain, qui existe en même temps dans le temps et l'espace. C'est ainsi que le corps du roi est tout autant régi par le temps que celui de Molière; les deux sont des figures historiques sorties de leur contexte spatio-temporel et replacées dans un contexte artistique. La culture et l'histoire se fondent dès lors dans le discours artistique de Wilson qui présente le souvenir (fragmentaire) comme une expérience (artistique).

Des bruits d'explosion accompagnent la danse du roi, ce qui présente la danse comme un champ de bataille et évoque quelque "danse macabre». Dans cette perspective, le roi lui-même peut être vu comme une figure symbolique de la mort qui vient chercher Molière. Le pouvoir royal devient alors pouvoir cosmique et les scènes se chargent d'une nouvelle perspective, universelle, qui dépasse les distinctions précédentes. Quand la mort entre dans l'image, toutes les oppositions précédentes deviennent un non-sens, 
car la mort est tout aussi indifférente aux êtres humains qu'à leur univers. Un autre conflit prend alors forme: celui qui oppose le point de vue de la mort au point de vue de l'humanité. À la lumière de ce conflit, la scène de danse institue deux niveaux fondamentaux: un niveau dramatique littéral, qui s'oppose à la situation de Molière, et un niveau symbolique, qui dépasse cette opposition et instaure un nouveau contexte.

Le mécanisme esthétique, qui consiste à créer une opposition au niveau dramatique littéral et à la nier en même temps au niveau symbolique ou interprétatif, est à l'œuvre dans l'ensemble de la vidéo, et en particulier dans les scènes de danse. D'un côté, les scènes de danse présentent le corps comme une entité vitale, puissante et active, par opposition au corps de Molière présenté comme une entité absente et impotente. De l'autre, toutes les scènes de danse offrent une représentation symbolique de la mort dans ses diverses relations au corps et à la vie. Cette analogie entre le corps dansant et le corps mourant ouvre une perspective ironique sur les oppositions binaires ainsi présentées.

\section{LA DANSE DES ACTEURS (section 3)}

Deux acteurs sont assis en face de Molière qui, assis dans son lit, accomplit à répétition divers mouvements de mains concertés. Suit une scène où les acteurs dansent dans le carré blanc. Ici, contrairement à la danse du roi, les acteurs ont un rapport direct avec Molière et leur danse est donc perçue comme une action qui fait suite à la scène précédente. Il est difficile de comprendre ce que fait exactement Molière sous le regard des acteurs. Mais à cause de la contiguité des deux scènes et du fait que les mains de Molière sont animées de mouvements manifestement contrôlés, j'ai pour ma part l'impression que lui aussi avec ses mains accomplit des mouvements de danse. Cette image des «mains qui dansent» apparaît à plusieurs reprises dans l'œuvre la plus chargée de sens étant sans doute la «danse de mains» rituelle, exécutée par les sœurs agenouillées, à la dernière séquence - et elle constitue un motif distinct. Mais dans cette scène, l'image des «mains qui dansent» s'oppose à celle de la danse des acteurs. On considère généralement les mains comme la partie la plus active et la plus créative du corps humain, c'est pourquoi la formule «mains qui dansent» connote la capacité d'action et de création de l'homme, qui renvoie au pouvoir de formuler l'identité, la vie et l'existence. Mais ici ce pouvoir est objet d'ironie: «les mains qui dansent", en tant que réduction du "corps qui danse», mettent plutôt l'accent sur l'incapacité que sur la capacité. Le pouvoir de l'homme est ainsi réduit à une ridicule incapacité d'agir ou d'influer sur les conditions mêmes de son existence. Vue sous cet angle, la présentation paradoxale de Molière dans une situation active et créative, mais dans un état d'invalidité et d'impuissance, constitue une distinction entre la corporéité et la spiritualité de l'existence humaine. Alors que dans le contexte de la danse du roi, Molière est présenté comme «Monsieur tout-le-monde», il est ici montré comme un artiste engagé dans un travail créateur. Mais dans les deux scènes, sa situation repose sur une fusion paradoxale de contradictions qui illustrent et magnifient la dualité de l'être et du non-être.

La chorégraphie de cette danse se fonde sur une série d'oppositions entre les deux danseurs: l'un est vêtu de blanc, l'autre de noir, et tandis que l'acteur vêtu de noir danse, l'acteur en blanc reste immobile, et vice versa. De plus, les évolutions de l'acteur en noir sont semblables à celles du roi: l'accent est mis sur la verticalité et le contrôle des mouvements conçus comme des transitions entre les postures. Par contraste, l'acteur en blanc présente une nouvelle forme de danse faite de mouvements rotatifs fluides tandis que le corps s'élève et se baisse. La danse forme une structure faite d'actions et de réactions et évoque les danses de compétition dans lesquelles un danseur essaie de prouver sa supériorité. Cette atmosphère de rivalité, combinée à la référence à la "danse macabre» du roi, introduit un niveau symbolique représentant la lutte entre la vie (le danseur en blanc) et la mort (le danseur en noir). Les éléments de leur danse contribuent à cette lutte en présentant le flux de la vie 
comme un combat perpétuel contre la mort, tandis que la mort, elle, est montrée comme une interruption de ce flux vital. Une fois de plus le niveau symbolique inscrit la situation dans un contexte universel qui tourne en dérision les oppositions instaurées au niveau littéral. Dans la lutte universelle contre la mort, rien n'est épargné, aucune action ou création humaine, ni corporéité ni spiritualité; tout devient nul et non avenu sous les assauts de la mort. Ironiquement cela vaut aussi pour la création de Wilson lui-même, car manifestement le Molière réel n'a rien à voir avec la vidéo de Wilson. En fait, il n'existe pas aujourd'hui de "Molière réel", mais plutôt une figure culturelle abstraite, construite à partir de divers fragments et de quelques objets. Encore une fois, Wilson y fait jouer une autoréférence ironique, qui remet en question son propre travail créateur et les matériaux dont il se sert.

\section{LA DANSE DU JEUNE GARÇON ET DE LA JEUNE FILLE}

La troisième scène de la section 5 présente un jeune garçon et une jeune fille habillés en noir et dansant dans le carré blanc. Leur danse, comme celle des acteurs, est basée sur une alternance d'actions et de réactions: le garçon fait bouger ses mains puis les immobilise dans une certaine position face à la jeune fille; elle réagit en faisant bouger ses mains autour des siennes, puis elle cesse et il réagit. Leurs corps gardent une position verticale statique; leurs mains sont la seule partie de leurs corps qui bouge. L'impression créée est celle d'un jeu d'enfants, mais le jeu de ces danseurs n'a pas le sens d'un jeu libre et joyeux. Ils semblent se comporter comme des marionnettes manipulées par un pouvoir occulte. Cette chorégraphie, qui ne touche que les mains, est ainsi associée aux «mains qui dansent» de Molière. De plus, tout comme les deux danses précédentes, cette danseci génère aussi une série d'oppositions entre le corps et les mains, le mouvement et la posture, la dynamique et la statique. Ces oppositions ont déjà été marquées, nous l'avons vu, comme une façon de représenter la distinction entre la vie et la mort, le fait d'être en vie et le fait de mourir: elles constituent donc un niveau symbolique. Mais ici le littéral et le symbolique fusionnent dans les mêmes mouvements de danse pour dépeindre la réification d'un être humain à travers la performance des «marionnettes animées». Leur danse présente ainsi une articulation artistique qui a perdu son pouvoir d'expressivité. Elle ne fait dès lors que prolonger et amplifier la précédente ironie sur le pouvoir de l'humanité, en présentant ici l'être humain comme un enfant se livrant à des jeux qui lui permettent de se donner l'illusion de la puissance et de la créativité: l'illusion, en fait, de la marionnette confrontée à de puissantes forces cosmiques.

Cette danse fait suite à deux scènes qui contiennent de nombreux plans de mains prises dans diverses situations ou actions. On remarque tout particulièrement les mains usées de Molière parcourant lentement son corps couché, en contraste avec les mains de son père, actives et pleines de vie, occupées à nettoyer le vieux fauteuil usé de Molière. Ce contraste renvoie à l'analogie déjà instituée entre la mort et le temps, où l'on peut voir s'affirmer le contrôle qu'exerce le temps sur le corps et la vie des humains. Mais on peut voir ici s'instaurer une nouvelle distinction entre une personne et un objet. Le temps exerce son pouvoir sur tout objet et sur toute créature de l'univers, mais si le père de Molière est capable d'en atténuer les effets sur le fauteuil usé, personne ne peut en faire autant pour Molière, pas même le docteur présenté précédemment. Dans cette optique, la danse des jeunes gens, fondée sur le mouvement des mains, s'inscrit dans le prolongement direct de cette série d'images, mais en inversant l'opposition qu'elles contenaient: les corps des danseurs, en effet, aussi jeunes, pleins de vie et d'allant soient-ils, n'en sont pas moins semblables au vieux corps usé et passif de Molière, parce qu'ils subissent la même mécanisation. Comme tout un chacun, Molière est réifié non seulement par la mort indifférente, mais aussi par la culture et l'art. À la mort, la culture humaine oppose le souvenir constitué à partir d'objets représentatifs qui remplacent la personne décédé. En ce sens, on peut dire qu'elle 
transforme du non-être en être. Mais ce faisant, elle consume aussi la personne et son œuvre. La vidéo de Wilson reflète ce courant culturel et y participe intentionnellement.

Certes la destruction de la création est représentée sous diverses formes tout au long de l'œuvre, mais elle devient l'image essentielle à partir de maintenant (section 5) et jusqu'à la dernière séquence. Dans la scène de danse suivante, qui est aussi la dernière (section 7), nous voyons un renard s'emparer du carré blanc et exercer son contrôle sur lui. Après quoi aucune autre performance artistique n'y prendra place, mais on pourra y voir, au contraire, Molière brûler ses œuvres. La séquence est centrée sur ses mouvements de mains: il ramasse les papiers et les jette dans le feu. L'image la plus forte de la scène est celle où Molière tente de laisser l'empreinte de sa main sur une page brûlée qui, bien entendu, tombe aussitôt en poussière. La danse du renard indique donc le triomphe final de la mort qui amène la destruction totale de la vie et de la création humaine.

En résumé, je dirai qu'à travers la corrélation établie entre le corps qui danse et d'autres images du corps, et en particulier celui de Molière, Wilson construit et déconstruit des relations interactives entre le corps naturel, le corps culturel et la corps artistique. Il institue donc une distinction entre l'identité historique de "Molière» et l'entité culturelle et la figure esthétique que recouvre ce même nom. Et pourtant, ironiquement, ce qui émerge de l'œuvre de Wilson (c'est-à-dire dans ce cadre esthétique) n'est ni naturel ni historique, ni même culturel au sens large du terme. En établissant ces distinctions, Wilson produit également une autoreprésentation de son propre travail, créant ainsi une «boucle déconstructrice».

Bien que la vidéo s'intitule La Mort de Molière et que sa fiction représente la vie et la mort de Molière, son sujet et son thème central semblent plutôt le propre univers esthétique de Wilson: un univers qui crée une confrontation entre l'art comme moyen de représenter la condition humaine et l'art comme une fin en soi, c'est-à-dire un univers esthétique détaché de la vie naturelle. De ce point de vue, la mort de Molière sert seulement à Wilson de moyen artistique pour présenter cette confrontation. Incontestablement Wilson exerce ainsi une certaine ironie sur lui-même et présente son acte de création comme le ferait un magicien, ne ramenant Molière à la vie que pour le tuer à nouveau, ces deux actions n'étant au demeurant que des actes appartenant à une fiction artistique. Ainsi la principale préoccupation de Wilson semble-t-elle d'illustrer la situation existentielle de l'artiste: aussi créatives et puissantes que soient ses images, elles ne parviennent jamais qu'à faire naître un univers de fiction. La pierre de touche de cette situation, c'est la mort. Bien que Wilson évoque l'horreur de la mort, la mort elle-même n'est jamais montrée que par des représentations symboliques, essentiellement dans les scènes de danse. Son travail artistique transforme l'horreur de la mort en une terreur esthétique sans effusion de sang. La vidéo se présente ainsi comme une descendance de l'esthétique de Racine, «l'inventeur de la terreur sans le sang» (section 2), une esthétique qui consiste à produire un sentiment de terreur par la représentation. Et c'est ainsi que les relations paradoxales entre le corps dansant et le corps mourant constituent une représentation artistique de la confrontation entre la mort naturelle - la terreur sanglante - et la présentation esthétique que fait Wilson de la mort: la terreur sans effusion de sang. On pourrait dire, paraphrasant les formulations du poème d'ouverture: «Ce n'est pas une vidéo sur la mort, la mort n'est pas un sujet de poésie... C'est une vidéo sur la mort... La vidéo observe la mort au travail...» 


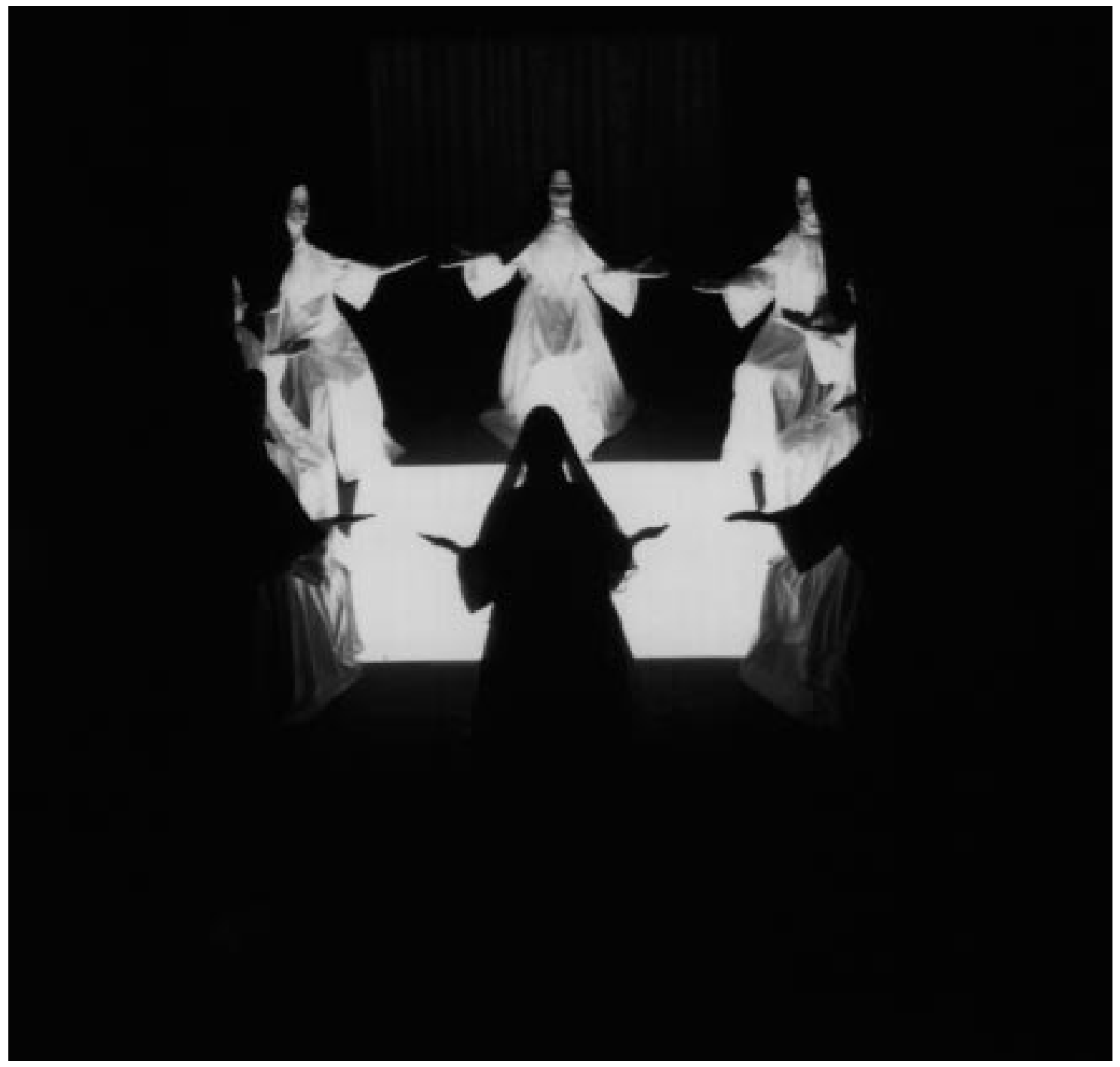

La Mort de Molière de Robert Wilson. Photo INA. 